\title{
Sorgfaltspflichten im Rahmen der Schwangerschaftsbetreuung
}

\author{
Eine Gynäkologin musste sich vor dem Oberlandesgericht München \\ verantworten. Ihr wurde vorgeworfen, eine Schwangerschaft fehlerhaft \\ überwacht und einen Kaiserschnitt zu spät durchgeführt zu haben.
}

m Jahr 2000 brachte die Gynäkologin an einem Montag ein bei der klagenden Kranken- und Pflegeversicherung versichertes Kind per Kaiserschnitt zur Welt. Bereits am Samstag und Sonntag stellte sich die Mutter des Kindes mit zunehmender Wehentätigkeit im Krankenhaus vor. Nach CTG-Aufzeichnung wurde sie jeweils wieder nach Hause entlassen. Die stationäre Aufnahme erfolgte am Montag gegen 1.00 Uhr. Nach wiederholter CTG-Aufzeichnung wurde der Frau gegen 9.30 Uhr ein wehenförderndes Mittel verabreicht. Um 9.40 Uhr ging sie spazieren, um 11.00 Uhr stellte sie sich mit zunehmenden Wehen erneut vor. Das von 11.02 Uhr bis 11.37 Uhr aufgezeichnete und von der Gynäkologin um 11.30 Uhr beurteilte CTG wies mäßig ausgeprägte Dezelerationen auf. Die Gynäkologin klärte die Mutter des Kindes über die Möglichkeit eines Kaiserschnitts auf. Die Geburt des Kindes erfolgte um 12.16 Uhr per Kaiserschnitt. Das Kind leidet an einer Hirnschädigung mit der Folge einer neurologischen Entwicklungsstörung.

Die Kranken- und Pflegeversicherung des Kindes warf der Gynäkologin mehrere Behandlungsfehler vor. So hätte die Mutter des Kindes am Wochenende nicht wieder nach Hause entlassen werden dürfen, zumal sie auch über ausbleibende Kindsbewegungen geklagt habe. Nach Gabe des wehenfördernden Mittels hätte sie kontinuierlich mittels CTG überwacht werden müssen. Schließlich sei der Kaiserschnitt zu spät erfolgt.

\section{So sah das Gericht den Fall}

Das Oberlandesgericht München hat sachverständig beraten - die Klage ab- gewiesen (Urteil vom 17.11.2011, Az. 24 U 675/09). Nach Ansicht des Sachverständigen sei das CTG bis zu der am Montag ab 11.02 Uhr erfolgten Aufzeichnung völlig unauffällig. Vor diesem Hintergrund hätten am Wochenende auch weder weitere Befunde erhoben noch irgendwelche Maßnahmen getroffen werden müssen. Hieran würde sich auch nichts ändern, wenn die Mutter des Kindes am Wochenende über ausbleibende Kindsbewegungen berichtet hätte. Denn dies könne ganz natürliche Ursache haben und die CTG-Werte hätten dafür gesprochen, dass es dem Kind gutgegangen sei. Im Übrigen seien zum Zeitpunkt der stationären Aufnahme der Mutter am Montag Kindsbewegungen festgestellt und auch dokumentiert worden.

Nach Gabe des wehenfördernden Mittels sei die seitens der Kläger geforderte kontinuierliche CTG-Überwachung nach Ansicht des Sachverständigen weder üblich, noch erforderlich und entspräche auch nicht dem ärztlichen Standard. Vielmehr genüge es, einer Schwangeren, die nach der Gabe des wehenfördernden Mittels nochmals etwas spazieren gehen wolle, aufzutragen, sich in Intervallen von ein bis zwei Stunden wieder zu melden. Diese Frist sei nicht überschritten worden.

Schließlich sei auch das weitere Handeln der Gynäkologin nach der gegen 11.30 Uhr erfolgten Feststellung der mäßig ausgeprägten Dezelerationen nicht zu beanstanden. Das CTG sei zu diesem Zeitpunkt als pathologisch, nicht aber als hochpathologisch zu bewerten. Nachdem kein Notfall vorgelegen habe, sei die Entscheidung zum

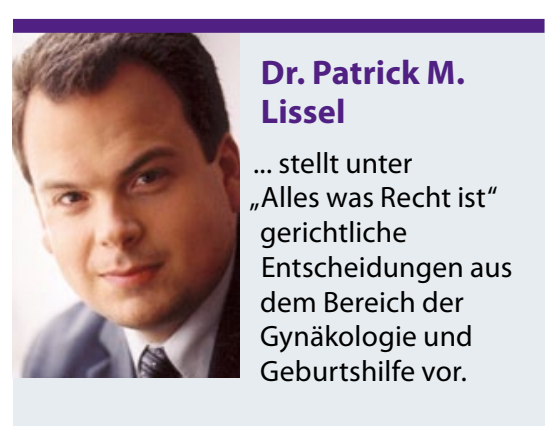

Kaiserschnitt auch nicht zu spät erfolgt. Eine Überlegungsfrist von ca. $20 \mathrm{Minu}$ ten sei nach dem vorliegenden CTGBefund als völlig angemessen anzusehen.

\section{Was bedeutet das Urteil für den klinischen Alltag?}

Der Sachverhalt zeigt neuerlich die erhebliche Bedeutung der Behandlungsdokumentation. Neben der Sorgfalt bei der Dokumentation ist auch auf die dauerhafte Lesbarkeit der Unterlagen $\mathrm{zu}$ achten. Vorliegend drohten die CTG-Aufzeichnungen zunehmend zu verblassen. Hierauf wurde das Gericht von Anfang an hingewiesen; die Originale wurden nebst zusätzlich angefertigter Kopien nach erfolgter Auswahl des Sachverständigen und Zustimmung des Gerichts ohne weitere Verzögerung direkt an den Sachverständigen verschickt. Es ist darauf zu achten, Originale, sofern deren Übermittlung im Ausnahmefall erforderlich ist, immer nur per Einschreiben/Rückschein zu versenden und zuvor für die eigenen Akten entsprechende Kopien anzufertigen.

\section{Dr. Patrick M. Lissel, LL.M.}

Rechtsanwalt und Fachanwalt für Medizinrecht

Ottostr. 1

80333 München 